\title{
OPEN A Chinese multicenter retrospective study of isolated increased nuchal translucency associated chromosome anomaly and prenatal diagnostic suggestions
}

\author{
Hua Jin ${ }^{1,8}$, Juan Wang ${ }^{2,8}$, Guoying Zhang ${ }^{3}$, Hongyan Jiao ${ }^{4}$, Jiansheng Zhu ${ }^{5}$, Zhimin $\mathrm{Li}^{2,6}$, \\ Chen $\mathrm{Chen}^{7}$, XuanPing Zhang ${ }^{2}$, Huan Huang ${ }^{3 \varpi}$ \& JiaYin Wang ${ }^{2 \bowtie}$
}

Extensive researches involving fetuses with multiple ultrasound anomalies have been conducted over the years, but only few were focused on the isolated increased nuchal translucency (NT). On top of that, these limited number of researches were all designed as single-arm studies and the control group was missing. In this study, we conducted a multicenter, retrospective study using amniotic fluid samples collected from 1197 pregnant women having fetuses with isolated increased NT (INT group) or normal NT values (NNT group). Copy number variation sequencing (CNV-seq) was performed to determine their chromosome status and pathogenic variations were validated using SNP array. Overall, 59 chromosome aneuploidies, 34 pathogenic CNVs and 23 copy number variants of unknown significance (VOUS CNVs) were discovered. the INT group had a significantly higher proportion of aneuploidy (19.44\%) and pathogenic CNV (8.33\%) than the control group (3.49\% and $2.30 \%$ respectively), and $\mathbf{8 8 . 8 9 \%}$ of the pathogenic CNVs were related to heart defects. Additionally, more male fetuses were presented in the INT group (68.51\%), but they did not have a higher risk (Relative Risk $=1.03$ ) of carrying pathogenic chromosome variations than female fetuses. Our results demonstrated that fetuses with isolated increased NT had a distinct pattern of chromosome abnormality and majority of detected pathogenic CNVs could be linked to the congenital heart disease. Furthermore, because a considerable proportion of pathogenic CNVs were detected, we strongly recommend to perform a joint test of karyotyping and CNV analysis in prenatal diagnosis for fetuses with isolated increased NT in order to decrease the incident of missed diagnosis.

For several decades, ultrasonography was widely used in prenatal diagnosis as a first-tier screening method and the thickness of nuchal translucency (NT) measured between $11^{+0}$ and $13^{+6}$ weeks in the first trimester was considered as an important indicator for Down syndrome ${ }^{1,2}$. In the clinical management guideline published by The American College of Obstetricians and Gynecologists (ACMG) and Society for Maternal Fetal Medicine, an increased NT is determined when the thickness of nuchal translucency is more than $3.0 \mathrm{~mm}$ or above the 99th percentile of the crown-rump length. The risk of trisomy 21 can be elevated to 1 in 46 when NT > 99th, whereas the risk was 1 in 8806 when $\mathrm{NT}<95 \mathrm{th}^{3}$. Hence, it is recommended that karyotyping should be conducted to confirm the chromosome status of fetuses with increased NT. Moreover, number of previous researches have

\footnotetext{
${ }^{1}$ Department of Prenatal Diagnosis, Ji'nan Maternal and Child Health Care Hospital, Ji'nan, Shandong, China. ${ }^{2}$ School of Computer Science and Technology, Xi'an Jiaotong University, Xi'an, Shaanxi, China. ${ }^{3}$ Department of Obstetrics and Gynecology, The First Affiliated Hospital of Nanjing Medical University, Nanjing, Jiangsu, China. ${ }^{4}$ Department of The Branch Center of Prenatal Diagnosis, Shijiazhuang Maternal and Child Care Service Hospital, Shijiazhuang, Hebei, China. ${ }^{5}$ Medical Genetic Center, Maternity and Child Health Hospital of Anhui Province, Affiliated Maternity and Child Health Hospital of Anhui Medical University, Anhui, China. ${ }^{6}$ Annoroad Gene Technology (Beijing) Co., Ltd, Beijing, China. ${ }^{7}$ Zhejiang Annoroad Biotechnology Co., Ltd, Zhejiang, China. ${ }^{8}$ These authors contributed equally: Hua Jin and Juan Wang. ${ }^{\boxplus}$ email: huanghuan@njmu.edu.cn; wangjiayin@mail.xjtu.edu.cn
} 


\begin{tabular}{|c|c|c|c|c|c|c|}
\hline \multirow[b]{2}{*}{ Anomalies } & \multicolumn{2}{|c|}{ NNT Group $(\mathrm{N}=1089)$} & \multicolumn{2}{|c|}{ INT Group $(\mathrm{N}=108)$} & \multirow[b]{2}{*}{$\mathbf{R R}^{\dagger}$} & \multirow[b]{2}{*}{$P$} \\
\hline & $\mathbf{N}$ & $\%$ & $\mathbf{N}$ & $\%$ & & \\
\hline Normal & 1004 & 92.19 & 77 & 71.30 & 0.77 & $<0.001^{* * *}$ \\
\hline Aneuploidy & 38 & 3.49 & 21 & 19.44 & 5.57 & $<0.001^{* * *}$ \\
\hline Pathogenic CNV & 25 & 2.30 & 9 & 8.33 & 3.62 & $<0.001^{* * x}$ \\
\hline VOUS CNV & 22 & 2.02 & 1 & 1.23 & 0.61 & 0.429 \\
\hline
\end{tabular}

Table 1. Incidence of aneuploidy and CNV in NNT and INT groups. Abbreviations: NNT: normal nuchal translucency; INT: increased nuchal translucency; CNV, copy-number variation; VOUS, variants of unknown significance; RR, relative risk. ${ }^{\dagger}$ Relative risk is calculated as \% cases of the anomaly in the INT Group/\% cases of the corresponding anomaly in the NNT Group, e.g. RR of aneuploidy: $19.44 \% / 3.49 \%=5.57$. The NNT group is used as the reference. ${ }^{\star * \star} \mathrm{P}<0.001$.

shown that copy number variation (CNV) could also be detected in fetuses with increased $\mathrm{NT}^{4-9}$ and the cause of increase by pathogenic CNVs (pCNVs) was through blood and lymph circulation disruption ${ }^{10}$.

Fetus with increased NT is a frequently observed symptom. While majority studies primarily concentrated on fetuses with multiple ultrasound indications, very few were focused on the ones with isolated increased NT. Previous publications demonstrated that on top of the increased incidents of chromosome aneuploidies. various percentages of pathogenic CNVs ranging from $0 \%$ to $12.8 \%{ }^{11-13}$ and high prevalence of congenital heart defects (CHD) and neurodevelopmental disorders were could also be found in fetuses with isolated increased $\mathrm{NT}^{14}$. Unfortunately, these researches were all designed as a single-arm study, and without a proper control group, results can only be compared with external historical data of other related work, which made it difficult to diminish the effects caused by different enrollment criteria among studies. In this work, we conducted a multicenter retrospective study with a control group to investigate chromosomal characteristics of fetuses with isolated increased NT and next-generation sequencing (NGS) based CNV-seq method was chosen to identify chromosome abnormalities because of its high sensitivity and specificity ${ }^{15,16}$. Our results showed that $8.33 \%$ of the fetuses in the isolated increased NT group carried pCNVs, which was significantly different $(P<0.001$, relative risk $=3.62$ ) than those in the normal NT group. Additionally, discrepancy in the distribution of pCNVs across chromosomes between the two groups was also observed and the risk of CHD was higher in fetuses with isolated increased NT. Furthermore, majority of cases in the isolated NT group were male (68.5\%, 74/108), but their relative risk of having chromosome anomalies was the same (relative risk=1.03) as female fetuses of the same group.

\section{Results}

Sample information. In this study, we analyzed 1197 amniotic fluid samples of high-risk Chinese pregnant women with singleton pregnancy. Samples were successfully tested using next-generation sequencing platform and they were all free of maternal contamination. Fetuses with normal NT and without any other ultrasound soft marker abnormalities was assigned as the normal NT group (NNT), whereas the ones with isolated increased NT was stratified as the isolated increased NT group (INT). The NNT group contained 1089 samples, the INT group had 108 cases and the mean maternal age was $32.43 \pm 5.71$ years and $30.19 \pm 4.89$ years respectively.

Detection of chromosomal abnormalities. Overall, a total of 116 anomalies was detected among 1197 samples, 59 (4.93\%) of which had chromosome aneuploidies, 34 (2.84\%) carried pathogenic CNVs and $23(1.95 \%)$ had copy number variants of unknown significance (VOUS CNVs). The INT group contained 21 $(19.44 \%, 21 / 108)$ aneuploidy cases, $9(8.33 \%, 9 / 108)$ pCNVs, $1(0.93 \%, 1 / 108)$ VOUS CNVs and $77(71.29 \%$, $77 / 108)$ normal cases, whereas the ratio was 3.49\% (38/1089), $2.30 \%(25 / 1089), 2.02 \%(22 / 1089)$ and $92.19 \%$ $(1004 / 1089)$ in the NNT group respectively (Table 1$)$. In comparison with the NNT group, the ratio of fetuses with normal karyotype, chromosomal aneuploidy and pCNV was significantly different in the INT group (all $P<0.001)$ and the relative risk of normal, aneuploidy and pCNV was $0.77,5.57$ and 3.62 respectively. The ratio of cases with VOUS CNVs, however, was not significantly different between the two groups $(P=0.429)$.

Comparison of chromosomal variations between NNT and INT group. The 108 samples in the INT group, can be further divided into two sub-groups on the basis of NT thickness (Table 2). In the 3-4 mm NT sub-group, the percentage of chromosomal aneuploidy, pathogenic CNV and VOUS CNV cases was $14.81 \%$ $(12 / 81), 7.41 \%(6 / 81)$ and $1.23 \%(1 / 81)$ respectively and it was $33.33 \%(9 / 27), 11.11 \%(3 / 27)$ and $0.00 \%(0 / 27)$ in the NT $>4 \mathrm{~mm}$ sub-group. Statistical analysis did not show any significant difference in the rate of pathogenic CNV and VOUS CNV between the two groups ( $P=0.546,0.562$ respectively). The $P$ value of chromosome aneuploidy was 0.035 , demonstrating a sign of increase with increased NT thickness. Moreover, the distribution of aneuploidy in the INT group was also different to that of the NNT group (Fig. 1a). In the INT group, trisomy 21 occupied $80.95 \%(17 / 21)$ of the aneuploidies and the rest of 4 cases were X0 (2/21, 9.52\%), trisomy 18 (1/21, $4.76 \%)$ and trisomy $22(1 / 21,4.76 \%)$. The anomalies in the NNT group, on the other hand, were spread out between trisomy $21(14 / 38,36.84 \%)$, trisomy X (8/38, 21.05\%), trisomy $18(5 / 38,13.16 \%)$, X0 (5/38, 13.16\%), $\mathrm{XYY}(5 / 38,13.16 \%)$ and trisomy $12(1 / 38,2.63 \%)$.

In terms of pathogenic CNVs, a total of 25 pCNVs in the NNT group and 9 pCNVs in the INT group were discovered (Table 3). One example of the pCNV detected by CNV-seq and SNP array were illustrated in Fig. 2. In the NNT group (Fig. 1b), 64\% of the pathogenic CNVs were located on chromosome 16 (5/25, 20.00\%), 


\begin{tabular}{|c|c|c|c|c|c|c|}
\hline \multirow[b]{2}{*}{ Anomalies } & \multicolumn{2}{|c|}{ NT 3-4 mm $(\mathrm{N}=81)$} & \multicolumn{2}{|c|}{$\mathrm{NT}>4 \mathrm{~mm}(\mathrm{~N}=27)$} & \multirow[b]{2}{*}{$\mathbf{R R}^{\dagger}$} & \multirow[b]{2}{*}{$P$} \\
\hline & $\mathbf{N}$ & $\%$ & $\mathbf{N}$ & $\%$ & & \\
\hline Normal & 62 & 76.54 & 15 & 55.56 & 0.73 & $0.037^{*}$ \\
\hline Aneuploidy & 12 & 14.81 & 9 & 33.33 & 2.25 & $0.035^{*}$ \\
\hline Pathogenic CNV & 6 & 7.41 & 3 & 11.11 & 1.50 & 0.546 \\
\hline VOUS CNV & 1 & 1.24 & 0 & 0 & 0 & 0.562 \\
\hline
\end{tabular}

Table 2. Incidence of aneuploidy and CNV discovered in NT 3-4 mm and > $4 \mathrm{~mm}$ sub-groups. Abbreviations: NT: nuchal translucency; CNV, copy-number variation; VOUS, variants of unknown significance; RR, relative risk. ${ }^{\dagger}$ Relative risk is calculated as \% cases of the anomaly in the NT $>4 \mathrm{~mm}$ group/\% cases of the corresponding anomaly in the NT $3-4 \mathrm{~mm}$ group, e.g. RR of aneuploidy: $33.33 \% / 14.81 \%=2.25$. NT $3-4 \mathrm{~mm}$ group is used as the reference. ${ }^{*} \mathrm{P}<0.05$.

\section{a)}

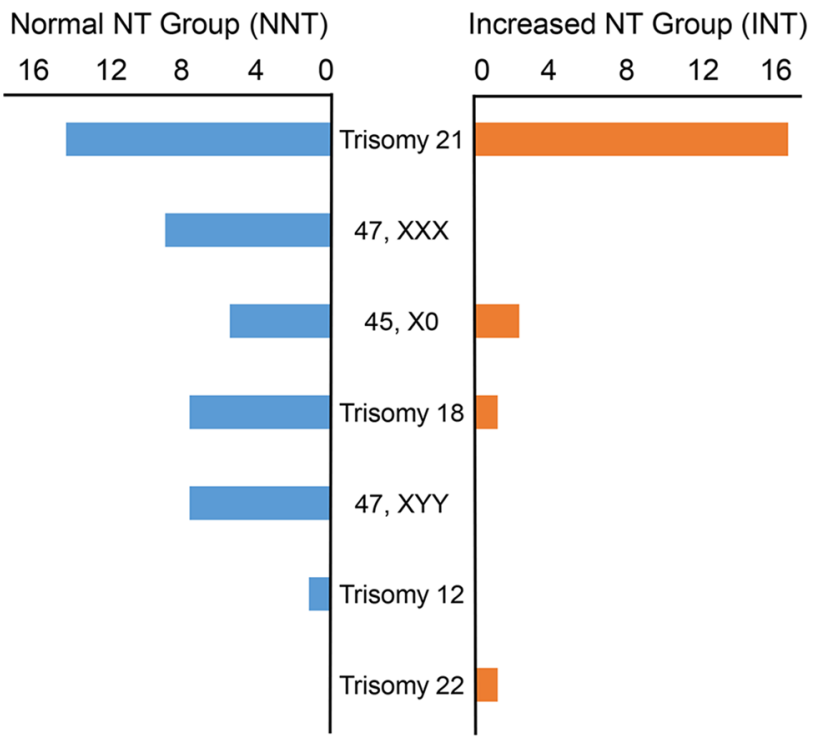

Chromosome Aneuploidy

b)

Normal NT Group (NNT) $\begin{array}{llllllll}7 & 6 & 5 & 4 & 3 & 2 & 1 & 0\end{array}$
Increased NT Group (INT) $\begin{array}{llllllll}0 & 1 & 2 & 3 & 4 & 5 & 6 & 7\end{array}$

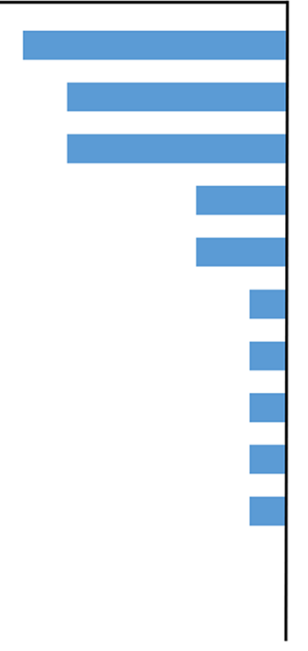

Chr. 22

Chr. 16

Chr. 17

Chr. 5

Chr. 8

Chr. 12

Chr. 15

Chr. 2

Chr. 3

Chr. X

Chr. 18

Chr. 6

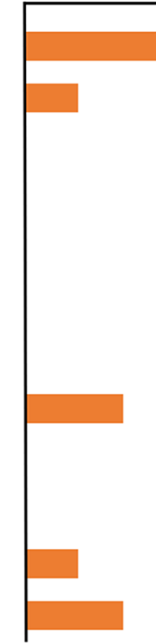

Pathogenic CNV

Figure 1. Distribution of chromosome aneuplodies and pathogenic CNVs in NNT and INT groups. (a) Number of chromosome aneuploidies showed distinct distribution patterns in NNT (blue) and INT groups (orange); (b) Number of pathogenic CNVs revealed that distribution of CNVs was very different between NNT (blue) and INT (orange) groups.

chromosome $17(5 / 25,20.00 \%)$ and chromosome $22(7 / 25,28.00 \%)$. The top 3 frequently observed pCNVs were $22 \mathrm{q} 11$ duplication syndrome $(5 / 25,20.00 \%), 16 \mathrm{p} 11.2$ duplication syndrome $(3 / 25,12.00 \%)$ and CharcotMarie-Tooth syndrome type $1 \mathrm{~A}(2 / 25,8.00 \%)$. In comparison, $77.78 \%$ of pCNVs in the INT group were located (Fig. 1b) on chromosome 2 (2/9, 22.22\%), $16(2 / 9,22.22 \%)$ and $22(3 / 9,33.33 \%)$, and 22q11 deletion syndrome (also known as the DiGeorge syndrome) had the highest occurrence rate $(3 / 9,33.33 \%)$.

\section{Discussion}

Increased NT detected by ultrasonography at $11^{+0}$ to $13^{+6}$ weeks is considered to be an effective indicator for fetal anomalies. Currently, it is used to predict up to $50 \%$ of major fetal abnormalities ${ }^{20}$ and it was associate with high levels of several adverse outcomes including miscarriage, fetal anomalies and genetic syndromes ${ }^{21-24}$. The mechanism of nuchal elevation is diversified. Cystic hygroma is a type of manifestation characterized by fluid-filled cavities (cysts) and it was found to be associated with trisomy 13, trisomy 18 and Turner's syndrome $(45, \mathrm{X} 0)$. The nuchal elevation exsited in non-45,X fetuses with cystic hygroma was caused by the proliferation of lymphatic vessels ${ }^{25,26}$. In Turner's syndrome, however, the primary cause of cystic hygroma is local aplasia of lymphatic vessels, which interrupts the drainage into the jugular lymph $\operatorname{sacs}^{27}$. By contrast, nuchal oedemas, a second type of NT elevation appearing as a non-echogenic area of over $3.5 \mathrm{~mm}$ below the skin and outside the cervical vertebral column in ultrasonic scans ${ }^{28}$, is closely associated with trisomy 21 . High level of precipitate around bundles of collagen fibrils and over-expression of collagen type VI in nuchal skin of fetuses could be observed $^{28,29}$. Significant increase of hyaluronan was also found in fetuses with trisomy 21 , suggesting that it may be involved in the pathogenesis of increased $\mathrm{NT}^{30}$. 


\begin{tabular}{|c|c|c|c|c|c|c|c|}
\hline Group & Sample ID & Fetus sex & CNV position ${ }^{\dagger}$ & Size $(\mathbf{M b})$ & Chromosomal disorder ${ }^{*}$ & Source of evidence & $\begin{array}{l}\text { Reported cases with CHD } \\
\text { and neurodevelopmental } \\
\text { disorders }^{\S}\end{array}$ \\
\hline NNT & 00433 & $\mathrm{~F}$ & chr2:g.10001_24135000dup & 24.13 & Partial trisomy $2 p$ & Lurie et al. ${ }^{43}$ & CHD, ID \\
\hline NNT & 00376 & $\mathrm{~F}$ & chr3:g.196965295_197415294dup & 0.50 & 3q29 duplication syndrome & $\# 611936$ & ID \\
\hline NNT & 00745 & F & chr4:g.10001_4015500del & 4.00 & Wolf-Hirschhorn syndrome & $\# 194190$ & CHD, ID \\
\hline NNT & 00806 & $\mathrm{M}$ & chr5:g.10001_11160000del & 11.15 & Cri du Chat Syndrome & $\# 123450$ & ID, rare CHD \\
\hline NNT & 00117 & $\mathrm{~F}$ & chr5:g.60001_6560000del & 6.50 & Cri du Chat Syndrome & $\# 123450$ & ID, rare CHD \\
\hline NNT & 00925 & M & chr8:g.12510001_23560000dup & 11.05 & 8p23.1 duplication syndrome & ORPHA:251076 & CHD, ID \\
\hline NNT & 00324 & M & chr12:g.60001_34810001dup & 34.75 & Trisomy 12p & ORPHA:1699 & CHD, ID \\
\hline NNT & 00823 & M & chr15:g.31976624_32526623del & 0.55 & 15q13.3 deletion syndrome & $\# 612001$ & ID, rare CHD \\
\hline NNT & 01229 & M & chr16:g.15460001_16310000dup & 0.85 & $\begin{array}{l}\text { 16p13.11 microduplication } \\
\text { syndrome }\end{array}$ & ORPHA:261243 & ID, rare CHD \\
\hline NNT & 00271 & M & chr16:g.25160001_26560000dup & 1.40 & $\begin{array}{l}\text { 16p11.2p12.2 microduplication } \\
\text { syndrome }\end{array}$ & ORPHA:261204 & rare ID, rare Schizophrenia \\
\hline NNT & 00702 & $\mathrm{~F}$ & chr16:g.29460001_30210000dup & 0.75 & 16p11.2 duplication syndrome & $\# 614671$ & ID; Schizophrenia \\
\hline NNT & 00692 & $\mathrm{~F}$ & chr16:g.29610001_30210000dup & 0.60 & 16p11.2 duplication syndrome & $\# 614671$ & ID, Schizophrenia \\
\hline NNT & 00319 & M & chr16:g.29610001_30160000del & 0.60 & 16p11.2 deletion syndrome & $\# 611913$ & ID, ASD \\
\hline NNT & 00234 & $\mathrm{M}$ & chr17:g.1_25400000dup & 25.40 & Trisomy $17 \mathrm{p}$ & ORPHA:261290 & ID, rare CHD \\
\hline NNT & 00300 & M & chr17:g.14050001_15500000dup & 1.45 & $\begin{array}{l}\text { Charcot-Marie-Tooth syndrome } \\
\text { type } 1 \mathrm{~A}\end{array}$ & $\# 118220$ & HMSNs \\
\hline NNT & 00457 & M & chr17:g.14100001_15500000dup & 1.40 & $\begin{array}{l}\text { Charcot-Marie-Tooth syndrome } \\
\text { type 1A }\end{array}$ & $\# 118220$ & HMSNs \\
\hline NNT & 01424 & $\mathrm{M}$ & chr17:g.16250001_22750000dup & 6.50 & Potocki-Lupski syndrome & $\# 610883$ & Autism, CHD, ID \\
\hline NNT & 01532 & M & chr17:g.34800001_36250000del & 1.45 & $\begin{array}{l}\text { Renal cysts and diabetes syn- } \\
\text { drome }\end{array}$ & \#137920 & rare ID \\
\hline NNT & 00837 & $\mathrm{~F}$ & chr22:g.18600001_21900004dup & 3.30 & 22q11 duplication syndrome & $\# 608363$ & ID, rare CHD \\
\hline NNT & 01069 & $\mathrm{~F}$ & chr22:g.18900001_21500004dup & 2.60 & 22q11 duplication syndrome & $\# 608363$ & ID, rare CHD \\
\hline NNT & 00593 & M & chr22:g.18900001_25150004dup & 6.25 & 22q11 duplication syndrome & $\# 608363$ & ID, rare CHD \\
\hline NNT & 00317 & F & chr22:g.18950001_2145000dup & 2.50 & 22q11 duplication syndrome & $\# 608363$ & ID, rare CHD \\
\hline NNT & 00952 & $\mathrm{~F}$ & chr22:g.20700005_21500004dup & 0.80 & 22q11 duplication syndrome & $\# 608363$ & ID, rare CHD \\
\hline NNT & 00042 & $\mathrm{~F}$ & chr22:g.18850001_21600004del & 2.75 & DiGeorge syndrome & $\# 188400$ & ADHD, CHD, rare Autism \\
\hline NNT & 00395 & M & chr22:g.45700005_51150004del & 5.45 & Phelan-McDermid syndrome & $\# 606232$ & rare ID \\
\hline INT & 10003 & M & chr2:g.61087852_61537851del & 0.45 & 2p16.1-p15 deletion syndrome & $\# 612513$ & ADHD, Autism, ID \\
\hline INT & 00608 & $\mathrm{M}$ & chr2:g.178879852_184979851del & 6.09 & 2q31.2 deletion syndrome & $\# 612345$ & ID, rare CHD \\
\hline INT & 00178 & M & chr6:g.88610001_100310000del & 11.7 & $\begin{array}{l}\text { Interstitial 6q microdeletion } \\
\text { syndrome }\end{array}$ & Vignoli et al..$^{44}$ & Autism, CHD, ID \\
\hline INT & 00305 & $\mathrm{~F}$ & chr6:g.128060001_171010000dup & 42.95 & Partial duplication of $6 \mathrm{q}$ & Conrad et al..$^{45}$ & CHD, ID \\
\hline INT & 00523 & $\mathrm{~F}$ & chr16:g.14860001_16660000dup & 1.80 & $\begin{array}{l}16 \mathrm{p} 13.11 \text { microduplication } \\
\text { syndrome }\end{array}$ & ORPHA:261243 & ADHD, CHD, ID \\
\hline INT & 00218 & $\mathrm{~F}$ & chr18:g.41460001_77210000dup & 35.75 & Partial trisomy $18 \mathrm{q}$ & Cereda et al. ${ }^{46}$ & CHD. ID \\
\hline INT & 00136 & F & chr22:g.18900001_21700004del & 2.80 & DiGeorge syndrome & $\# 188400$ & ADHD, CHD, rare Autism \\
\hline INT & 00936 & M & chr22:g.18900001_21500004del & 2.60 & DiGeorge syndrome & $\# 188400$ & ADHD, CHD, rare Autism \\
\hline INT & 00451 & $\mathrm{M}$ & chr22:g.18800001_21700004del & 2.90 & DiGeorge syndrome & $\# 188400$ & ADHD, CHD, rare Autism \\
\hline
\end{tabular}

Table 3. The list of pathogenic CNVs identified in the normal NT and enlarged NT groups. Abbreviations: NNT, normal NT; INT, increased NT; M, male; F, female; CNV, copy-number variation; ORPHA, Orphanet database; ADHD, attention deficit hyperactivity disorder; ASD, autism spectrum disorder; CHD, congenital heart disease; HMSNs, hereditary motor and sensory neuropathies; ID, intellectual disability. ${ }^{\dagger}$ The positions of CNV are written in accordance with the International System for Human Cytogenomic Nomenclature (ISCN, 2016). ${ }^{\ddagger}$ The name of the disorder is written as the entry name of OMIM (Online Mendelian Inheritance in Man) database, Orphanet database or descriptions in the cited articles. ${ }^{\S}$ Symptom description of the corresponding pathogenic CNV was obtained from OMIM database, Orphanet database or cited articles.

Different from previous researches, we focused on the fetuses with isolated increased NT in this work. By doing so, we could then investigate their molecular characteristics and provide a strong basis for guiding the choice of subsequent diagnostic strategies in clinical settings. Overall, we analyzed and compared chromosomeal variations between NNT control group (1089 samples) and INT group (108 samples). For chromosome aneuploidy, previous researches demonstrated that the rate of aneuploidy was positively correlated with increased NT thickness. The percentage of chromosomal defects was 3.7\% when NT value was between $2.5-3.5 \mathrm{~mm}$ and it was increased to $21.1 \%$ or above $60 \%$ when the NT thickness reached $3.5-4.5 \mathrm{~mm}$ or over $6.5 \mathrm{~mm}$ respectively ${ }^{12}$. In our work, we also demonstrated a significant increase in the percentage of aneuploidies and the incident rate was increased from $14.81 \%$ to $33.33 \%$ in NT $3.0-4.0 \mathrm{~mm}$ and NT $>4.0 \mathrm{~mm}$ subgroups. Moreover, the differences 
a) 3

2 Copy Number 1

0

512

384

256

128

Depth

0

\section{Chromosome 22}
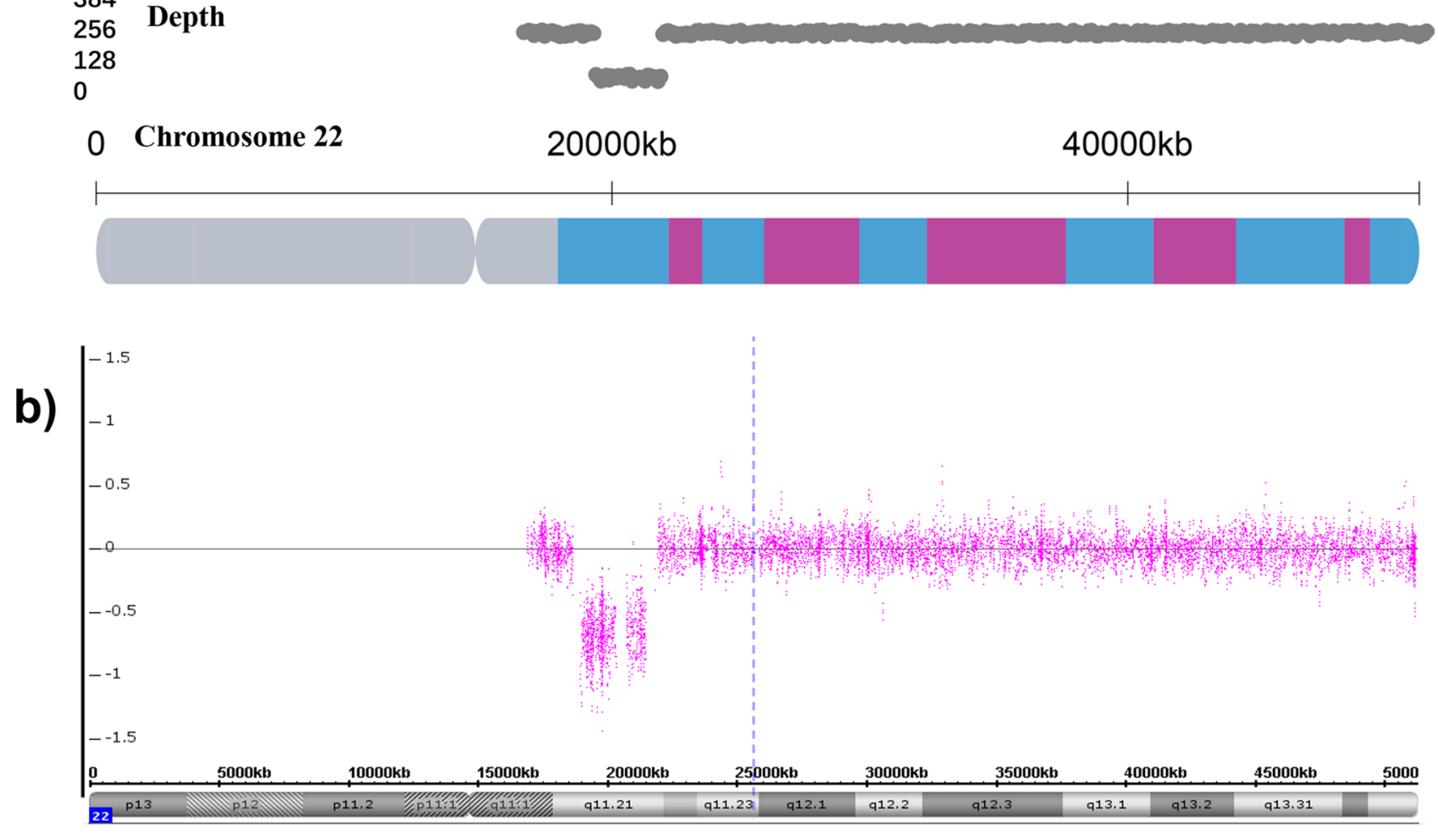

Figure 2. CNV-seq analysis and SNP array confirmation of the fetus with Di George syndrome (sample ID: 00936). (a) CNV-seq analysis showed the presence of 22q11 deletion (green) in the amniotic fluid sample; (b) $\mathrm{SNP}$ array validated the $22 \mathrm{q} 11$ deletion in the fetus.

in the distribution of aneuploidy between the NNT and INT groups suggested that the increased NT value was closely associated with trisomy 21 , whereas aneuploidies of $47, \mathrm{XXX}$ and $47, \mathrm{XYY}$ were less likely to cause the elevation.

Unlike chromosome aneuploidy, CNVs were frequently found in human genomes ${ }^{31}$ and based on its influence to human health, it is classified as benign, VOUS and pathogenic. Since the clinical significance of VOUS CNVs is not clear, we only focused on assessing the relationship between pathogenic CNVs in this work. The potential biological effects of pathogenic CNVs on the relevant diseases were believed to be related to the deletion or duplication of morbid genes. For example, Kerzendorfer et al. ${ }^{32}$ Discovered that deletion of SLBP or NELFA genes may contribute to the clinical features of Wolf-Hirschhorn syndrome, such as growth retardation and microcephaly. Both of these genes are involved in histone biogenesis and all patient cell lines with variable deletion showed delayed progression from S-phase to M-phase of the cell cycle and defective DNA replication and reduced levels of chromatin-associated histones after DNA replication were also observed. Medina et al. ${ }^{33}$ Demonstrated that the CTNND2 gene was closely related to the mental retardation phenotype of cri-du-chat syndrome by showing the strong correlation between hemizygous loss of CTNND2 and severe mental retardation. CTNND2 is a neuronal specific protein and expressed during early development and involved in cell motility. These properties may support its role in the mental retardation of the syndrome when presented in only one copy. Moreover, micro RNA (MIR) also seem to be associated with CNV related disease. Weber et al. Closely examined a case of 8p23.1 duplication syndrome through SNP array analysis, he found that dosage sensitive genes such as SOX7, TNKS1, MIR124-1 and MIR598 were located in the core duplicated interval. Both MIR124-1 and MIR598 genes have been implicated in neuropsychiatric disorders and so he suggested that these two MIRs might be a contributing factor to autism spectrum disorder in the $8 \mathrm{p} 23.1$ duplication syndrome patient ${ }^{34}$. Finding the morbids genes, however, is a difficult and ongoing task because the deleted or duplicated region may contain many genes. For example, 3q29 duplication syndrome is a CNV related disease, most patients have eye abnormalities, intellectual disability and small head. It has an additional copy of $1.6 \mathrm{Mb}$ at position 29 on chromosome 3 and this duplicated segment contains about 20 genes. Although some of these genes are thought to be involved in brain and eye development, it is still unknown which specific genes, when abnormally copied, are related to the varied signs and symptoms of the syndrome. 
In this work, our data showed that the percentage of pCNVs detected in fetuses in the INT group was $8.33 \%$, which was consistent to the previously reported range of $6.86 \%$ to $9.09 \%$ in NT $>99$ th samples ${ }^{12,35}$, and it was significantly higher than that of the NNT group. However, no significant difference in pCNV incident rate was found between $3.0-4.0 \mathrm{~mm}$ and $>4 \mathrm{~mm}$ sub-groups and this suggested that, unlike chromosome aneuploidy, the occurrence of pathogenic CNVs would not increase further when NT value was above $3 \mathrm{~mm}$. In addition, previous literatures reported that fetus with increased NT may have an increased risk of $\mathrm{CHD}^{4}$ and different hypotheses on the aetiology of the increased NT have been suggested ${ }^{36}$. Since our enrollment criteria excluded fetuses with other ultrasound anomalies including heart defects, we therefore looked into potential symptoms which could be caused by the identified pathogenic CNVs. The description of symptoms were collected from OMIM (Online Mendelian Inheritance in Man) and ORPHANET database, whereas the rest of information was obtained from appropriate literatures.

In our data, the most common pathogenic CNVs detected in the INT group was Di George syndrome, which was well-known to cause malformation of the heart. Other pCNVs, such as chromosome $2 \mathrm{q} 31.2$ deletion syndrome, $6 \mathrm{q}$ microdeletion syndrome, partial 6q duplication, $16 \mathrm{p} 13.11$ microduplication syndrome and partial trisomy 18q, of the same group were also reported to cause heart defects with the exception of chromosome 2 p16.1-p15 deletion syndrome $(1 / 9,11.11 \%)$. In contrast, only 9 out of 25 pathogenic CNVs in the NNT group, namely partial trisomy 2p, Wolf-Hirschhorn syndrome, 8 p23.1 duplication syndrome, trisomy 12p, chromosome $15 q 13.3$ deletion syndrome, 16p13.11 microduplication syndrome, Trisomy 17p, Potocki-Lupski syndrome and Di George syndrome, could be linked to venticular septal defect, atrial septal defect or hypoplastic left heart, whereas the rest of $16(64 \%)$ pCNVs have not yet established a clear association to CHD. What's more, difference in types of pCNVs was also discovered between the two groups, $68.00 \%(17 / 25)$ of pCNVs in the NNT group had microduplications, whereas $66.67 \%(6 / 9)$ of pCNVs in the INT group were microdeletions. However, due to the limited number of samples, this finding needs to be further validated using larger cohort and its potential implications also need to be investigated. Furthermore, it was reported that the gender of fetus may also play a role in the increased NT thickness ${ }^{37}$ and we have investigated this claim in our work. The results showed that $68.51 \%$ (74/108) of the INT group were male fetuses and 31.48\% (34/108) were female fetuses which was in concordance with the previous finding. However, the ratio of pathogenic chromosome anomalies in males and females was almost identical $(P=0.94), 24.32 \%(18 / 74)$ and $23.53 \%(8 / 34)$ respectively. Male fetuses did not have higher risk $(\mathrm{RR}=1.03$, female is used as the reference $)$ of carrying pathogenic chromosome variations than female fetuses.

Despite our best efforts to distinguish nuchal hygroma from nuchal oedemas using ultrasonography, we could not completely rule out the possible inclusion of nuchal hygroma samples, which is a limitation of this study. Although the cases of trisomy 18 and Tuner sydrome in the INT group showed no signs of cysts under ultrasound examination, miscarriage tissues were not collected and examined and so the exact nature of the NT elevation was undetermined. Also, this work only focused on the effects of chromosomal aneuploidy and copy number variation on the increased NT, which was mainly caused by incorrect seperation of chromosome or chromosome lost.

during the maturation process of germ cells or early cleavage of fertilized eggs and non-allelic homologous recombination respectively. Environmental factors such as malnutrition may also associate with heart or neural abnormal development, but it is not examined in this work. According to previous literatures, prenatal malnutrition might have an impact on genetic selection, which would increase the chance of obtaining de novo genetic mutations in germ cells and transmit to the offspring if the starvation occurs before fertilization or elevate the probability of passing on the alleles of neuropsychiatric disorders to the next generation when pregnant women under nutritional stress ${ }^{38,39}$. In addition, transcriptome analysis using mouse model under prenatal nutritional deficient environment revealed altered gene expression profile and discovered 15 key genes related to autism and schizophrenia ${ }^{40-42}$.

In summary, NT thickness was originally proposed to evaluate the risk of Down syndrome and fetuses with isolated increased NT anomaly are usually diagnosed with the karyotyping alone, but we demonstrated in this work that fetuses with isolated increased NT also had additional $8.33 \%$ of pathogenic CNVs on top of chromosome aneuploidies, and $88.89 \%$ of which appeared to be associated with heart defects. Although no signs of CHD were discovered by the ultrasound throughout the entire pregnancy, these fetuses with pCNVs would be highly likely to develop severe symptoms during infancy or childhood. Therefore, it is most beneficial to integrate CNV analysis and karyotyping in prenatal diagnosis to avoid missed diagnosis.

\section{Material and methods}

Sample collection. This was a joint retrospective study with prenatal diagnosis centers across four Chinese provinces Anhui, Jiangsu, Shandong and Shannxi. The enrollment criteria was: 1 . women underwent amniocentesis procedure between $16+0$ to $18+6$ weeks; 2 . singleton pregnancy; 3 . pregnant women without inheritable risk, tumor, pre-eclampsia, and prior risk of abnormal pregnancy outcome; 4 . crown-rump length was between 45 and $84 \mathrm{~mm}$; 5 . NT was measured between $11+0$ and $13+6$ weeks; 6 . fetuses without any ultrasound anomalies (NNT group); 7. fetuses with increased NT (INT group) and no other ultrasound soft marker abnormalities (e.g. nuchal hygroma, mild ventricular expansion, intracardiac echogenic focus, hyperechogenic bowel, absence or dysplasia of nasal bone, short long bones, single umbilical artery, choroid plexus cyst, increased cisterna magna and hydronephrosis etc.); 8. CNV-seq analysis was carried out using amniotic fluids; 9. pathogenic CNVs detected by CNV-seq were confirmed by CMA.

In total, 1197 pregnant women between January 2017 and March 2019 were enrolled, their archived clinical records and sequencing results were retrieved from the Jinan Maternal and Child Health Care Hospital, the First Affiliated Hospital of Nanjing Medical University, the Shijiazhuang Maternal and Child Care Service Hospital and the Maternity and Child Health Hospital of Anhui Province, Affiliated Maternity and Child Health Hospital of 
Anhui Medical University. Pregnant women were stratified into 2 categories on the basis of the NT thickness, the normal NT group (NNT group) and the isolated increased NT group (INT group). The NNT group consists of fetuses with the NT value less than $3 \mathrm{~mm}$. The INT group, on the other hand, contained fetuses with NT $\geq 3 \mathrm{~mm}$.

For 59 pregnant women with chromosome aneuploidies, 9 of which experienced miscarriage, 43 had induced abortion, and 7 with fetuses carrying 47, XYY or 47, XXX continued their pregnancy and gave birth. Six pregnant women with cases of DiGeorge syndrome and Cri du Chat Syndrome chose to take induced abortion, whereas the rest of CNV cases continued their pregnancy and gave birth to a living infant.

CNV-seq. CNV-seq was performed by following manufacture's protocol. In short. total genomic DNA was isolated from amniotic fluid samples using the Amp Genomic DNA Kit (TIANGEN Biotech, Beijing, China) according to the user's manual. Next generation sequencing was performed as previously described ${ }^{17}$. In short, genomic DNA was fragmented to the average size of $200 \mathrm{bp}$ and $2.5 \mathrm{ng}$ of fragmented DNA was used for the sequencing library construction. Barcoded sequencing adaptors were ligated to the DNA fragments and amplified by the polymerase chain reaction (PCR). The PCR product was then purified using magnetic beads and the constructed libraries were pooled and sequenced with NextSeq 550AR platform (Annoroad Technology, China). Finally, 8-10 million of 35 bp single-end raw reads were generated for each sample.

After sequencing quality control and trimming, short reads were aligned to the human reference genome (hg19) with the BWA aligner. Unique reads were counted for each of the $100 \mathrm{~kb}$ window. GC bias of per window read counts was corrected using the LOWESS model. The normal-karyotype database (NKD), derived from a group of 1000 samples with normal karyotype confirmed by G-banded karyotype analysis, was used as the background. Algorithms used for the bioinformatics analysis was detailed in the previous literature ${ }^{18}$. According to the recommendations of the ACMG standards and guidelines ${ }^{19}, \mathrm{CNV}$ s detected by the analysis were classified as pathogenic, variants of unknown significance (VOUS) or benign. Benign CNVs was usually considered as population polymorphism, thus samples with benign CNVs were classified as normal in this study.

CMA validation. SNP array was used in all four institutes to verify the pathogenic CNVs identified by the CNV-seq analysis. Briefly, Affymetrix Genechip CytoScan 750 K SNP Array (ThermoFisher, Shanghai, China) was used according to the user's manual for the CNV identification with the average inter probe distance of $100 \mathrm{~kb}$. The accompanied data analysis software Chromosome Analysis Suite (ChAS, version 1.2.1, ThermoFisher, https://www.thermofisher.com/cn/zh/home/life-science/microarray-analysis/microarray-analy sis-instruments-software-services/microarray-analysis-software/chromosome-analysis-suite.html) was used to calculate the $\log 2$ intensity ratio.

Statistical analysis. The differences between groups were examined using the chi-square test by Statistical Product and Service Solutions software (SPSS, version 22, IBM, https://www.ibm.com/analytics/spss-statistics -software). A significant P value was defined as 0.05 .

Ethical approval. This study was approved by the institutional ethics committee of each hospital, including the Jinan Maternal and Child Health Care Hospital, the First Affiliated Hospital of Nanjing Medical University, the Shijiazhuang Maternal and Child Care Service Hospital and the Maternity and Child Health Hospital of Anhui Province, Affiliated Maternity and Child Health Hospital of Anhui Medical University, with the exemption of patient's informed consent because only retrospective data was used. All experiments were performed in accordance with relevant regulations and details.

\section{Data availability and material}

Sequence data of this study are available upon reasonable request.

Received: 23 April 2020; Accepted: 15 February 2021

Published online: 10 March 2021

\section{References}

1. Malone, F. D. et al. First-trimester or second-trimester screening, or both, for Down's syndrome. N. Engl. J. Med. 353, 2001-2011 (2005).

2. Nicolaides, K. H., Azar, G., Snijders, R. J. \& Gosden, C. M. Fetal nuchal oedema: associated malformations and chromosomal defects. Fetal Diagn. Ther. 7(2), 123-131 (1992).

3. Hellmuth, S. G. et al. Increased nuchal translucency thickness and risk of neurodevelopmental disorders. Ultrasound Obstet. Gynecol. 49, 592-598 (2017).

4. Brady, P. D. et al. A prospective study of the clinical utility of prenatal chromosomal microarray analysis in fetuses with ultrasound abnormalities and an exploration of a framework for reporting unclassified variants and risk factors. Genet. Med. 16, 469-476 (2014).

5. Evangelidou, P. et al. Implementation of high resolution whole genome array CGH in the prenatal clinical setting: advantages, challenges, and review of the literature. Biomed. Res. Int. 2013, 346762 (2013).

6. Hillman, S. C. et al. Use of prenatal chromosomal microarray: prospective cohort study and systematic review and meta-analysis. Ultrasound Obstet. Gynecol. 41, 610-620 (2013).

7. Faas, B. H. et al. Non-targeted whole genome $250 \mathrm{~K} \mathrm{SNP}$ array analysis as replacement for karyotyping in fetuses with structural ultrasound anomalies: evaluation of a one-year experience. Prenat. Diagn. 32, 362-370 (2012).

8. Srebniak, M. I. et al. Genomic SNP array as a gold standard for prenatal diagnosis of foetal ultrasound abnormalities. Mol. Cytogenet. 5, 14 (2012).

9. Rooryck, C. et al. Prenatal diagnosis using array-CGH: a French experience. Eur. J. Med. Genet. 56, 341-345 (2013). 
10. Clur, S. A., Ottenkamp, J. \& Bilardo, C. M. The nuchal translucency and the fetal heart: a literature review. Prenat. Diagn. 29, 739-748 (2009).

11. Huang, J. et al. Is high fetal nuchal translucency associated with submicroscopic chromosomal abnormalities on array CGH? Ultrasound Obstet. Gynecol. 43, 620-624 (2014).

12. Lund, I. C., Christensen, R., Petersen, O. B., Vogel, I. \& Vestergaard, E. M. Chromosomal microarray in fetuses with increased nuchal translucency. Ultrasound Obstet. Gynecol. 45, 95-100 (2015).

13. Leung, T. Y. et al. Identification of submicroscopic chromosomal aberrations in fetuses with increased nuchal translucency and apparently normal karyotype. Ultrasound Obstet. Gynecol. 38, 314-319 (2011).

14. Egloff, M. et al. Diagnostic yield of chromosomal microarray analysis in fetuses with isolatedincreased nuchal translucency: a French multicenter study. Ultrasound Obstet. Gynecol. 52, 715-721 (2018).

15. Dong, Z. et al. Low-pass whole-genome sequencing in clinical cytogenetics: a validated approach. Genet. Med. 18, 940-948 (2016).

16. Liang, D. et al. Copy number variation sequencing for comprehensive diagnosis of chromosome disease syndromes. J. Mol. Diagn. 16, 519-526 (2014).

17. Liang, D. et al. Non-invasive prenatal testing of fetal whole chromosome aneuploidy by massively parallel sequencing. Prenat. Diagn. 33, 409-415 (2013).

18. Qi, H. et al. High resolution global chromosomal aberrations from spontaneous miscarriages revealed by low coverage whole genome sequencing. Eur. J. Obstet. Gynecol. Reprod. Biol. 224, 21-28 (2018).

19. Kearney, H. M. et al. American College of Medical Genetics standards and guidelines for interpretation and reporting of postnatal constitutional copy number variants. Genet. Med. 13, 680-685 (2011).

20. Rossi, A. C. \& Prefumo, F. Accuracy of ultrasonography at 11-14 weeks of gestation for detection of fetal structural anomalies: a systematic review. Obstet. Gynecol. 122, 1160-1167 (2013).

21. Senat, M. V. et al. Pregnancy outcome in fetuses with increased nuchal translucency and normal karyotype. Prenat. Diagn. 22, 345-349 (2002).

22. Souka, A. P., Von Kaisenberg, C. S., Hyett, J. A., Sonek, J. D. \& Nicolaides, K. H. Increased nuchal translucency with normal karyotype. Am. J. Obstet. Gynecol. 192, 1005-1021 (2005).

23. Baer, R. J. et al. Risk of selected structural abnormalities in infants after increased nuchal translucency measurement. Am. J. Obstet. Gynecol. 211(675), e1-19 (2014).

24. Tiyatha, S., Sirilert, S., Sekararithi, R. \& Tongsong, T. Association between unexplained thickened nuchal translucency and adverse pregnancy outcomes. Arch. Gynecol. Obstet. 298, 97-101 (2018).

25. Chitayat, D., Kalousek, D. K. \& Bamforth, J. S. Lymphatic abnormalities in fetuses with posterior cervical cystic hygroma. Am. J. Med. Gen. 33, 352-356 (1989).

26. von Kaisenberg, C. S. et al. Morphological classification of nuchal skin in fetuses with trisomy, 21, 18 and 13 at $12-18$ weeks and in a trisomy 16 mouse. Anat. Embryol. 197, 105-124 (1998).

27. Brand-Saberi, B. et al. Alterations of the fetal extracellular matrix in the nuchal oedema of Down's syndrome. Ann. Anat. 176, 539-547 (1994).

28. Brand-Saberi, B., Epperlein, H. H., Romanos, G. E. \& Christ, B. Distribution of extracellular matrix components in nuchal skin from fetuses carrying trisomy 18 and trisomy 21. Cell Tissue Res. 277, 465-475 (1994).

29. von Kaisenberg, C. S. et al. Collagen type VI gene expression in the skin of trisomy 21 fetuses. Obstet. Gynaecol. 91, 319-323 (1998).

30. Böhlandt, S. et al. Hyaluronan in the nuchal skin of chromosomally abnormal fetuses. Hum. Reprod. 15(5), 1155-1158 (2000).

31. Zarrei, M., MacDonald, J. R., Merico, D. \& Scherer, S. W. A copy number variation map of the human genome. Nat. Rev. Genet. 16, 172-183 (2015).

32. Kerzendorfer, C. et al. Characterizing the functional consequences of haploinsufficiency of NELF-A (WHSC2) and SLBP identifies novel cellular phenotypes in Wolf-Hirschhorn syndrome. Hum. Mol. Genet. 21, 2181-2193 (2012).

33. Medina, M., Marinescu, R. C., Overhauser, J. \& Kosik, K. S. Hemizygosity of delta-catenin (CTNND2) is associated with severe mental retardation in cri-du-chat syndrome. Genomics 63, 157-164 (2000).

34. Weber, A., Köhler, A., Hahn, A. \& Müller, U. 8p23.1 duplication syndrome: narrowing of critical interval to $1.80 \mathrm{Mbp}$. Mol. Cytogenet. 7, 94 (2014).

35. Pérez, S. P. Contribution of chromosomal microarray analysis in fetuses with increased nuchal translucency: a prospective observational study. Gynecol. Obstet. (Sunnyvale) 8, 479 (2018).

36. Haak, M. C. \& van Vugt, J. M. Pathophysiology of increased nuchal translucency: a review of the literature. Hum. Reprod. Update 9, 175-184 (2003).

37. Timmerman, E., Pajkrt, E. \& Bilardo, C. M. Male gender as a favorable prognostic factor in pregnancies with increased nuchal translucency. Ultrasound Obstet. Gynecol. 34, 373-378 (2009).

38. St Clair, D. et al. Rates of adult schizophrenia following prenatal exposure to the Chinese famine of 1959-1961. JAMA 294, 557-562 (2005).

39. Xu, M. et al. Prenatal malnutrition and adult schizophrenia: further evidence from the 1959-1961 Chinese famine. Schizophr. Bull. 35, 568-576 (2009).

40. Chen, J., Zhao, X., Cui, L., He, G. \& Xu, M. Genetic regulatory subnetworks and key regulating genes in rat hippocampus perturbed by prenatal malnutrition: implications for major brain disorders. Aging (Albany NY). 12, 8434-8458 (2020).

41. Li, H., Wang, X., Lu, X., Zhu, H. \& Xu, M. Co-expression network analysis identified hub genes critical to triglyceride and free fatty acid metabolism as key regulators of age-related vascular dysfunction in mice. Aging (Albany NY). 11, 7620-7638 (2019).

42. Yan, X., Zhao, X., Li, J., He, L. \& Xu, M. Effects of early-life malnutrition on neurodevelopment and neuropsychiatric disorders and the potential mechanisms. Prog. Neuropsychopharmacol. Biol. Psychiatry 83, 64-75 (2018).

43. Lurie, I. W. et al. Trisomy 2p: analysis of unusual phenotypic findings. Am. J. Med. Genet. 55, 229-236 (1995).

44. Vignoli, A., Scornavacca, G. F., Peron, A., La Briola, F. \& Canevini, M. P. Interstitial 6q microdeletion syndrome and epilepsy: a newpatient and review of the literature. Am. J. Med. Genet. A. 161A, 2009-2015 (2013).

45. Conrad, B. A., Higgins, R. R. \& Pierpont, M. E. Duplication 6q22->qter: definition of the phenotype. Am. J. Med. Genet. 78, $123-126$ (1998).

46. Cereda, A. \& Carey, J. C. The trisomy 18 syndrome. Orphanet. J. Rare Dis. 7, 81 (2012).

\section{Author contributions}

H.H. and J.W. laid out the overall study design. Data collection and analysis were performed by H.J., G.Z., H.J., and J.Z. Data analysis was done by J.W., Z.L., C.C. and X.Z. The first draft of the manuscript was written by H.J. and J.W., and all authors commented on previous versions of the manuscript. All authors read and approved the final manuscript.

\section{Funding}

The funding of this study is provided by the National Key R\&D Program of China (Grant number: 2016YFC1000307-12). 


\section{Competing interests}

The authors declare no competing interests.

\section{Additional information}

Correspondence and requests for materials should be addressed to H.H. or J.W.

Reprints and permissions information is available at www.nature.com/reprints.

Publisher's note Springer Nature remains neutral with regard to jurisdictional claims in published maps and institutional affiliations.

(c) (i) Open Access This article is licensed under a Creative Commons Attribution 4.0 International License, which permits use, sharing, adaptation, distribution and reproduction in any medium or format, as long as you give appropriate credit to the original author(s) and the source, provide a link to the Creative Commons licence, and indicate if changes were made. The images or other third party material in this article are included in the article's Creative Commons licence, unless indicated otherwise in a credit line to the material. If material is not included in the article's Creative Commons licence and your intended use is not permitted by statutory regulation or exceeds the permitted use, you will need to obtain permission directly from the copyright holder. To view a copy of this licence, visit http://creativecommons.org/licenses/by/4.0/.

(c) The Author(s) 2021 